\title{
Posibilidades y límites de la intervención pública dirigida a personas LGBT+ en Chile
}

\section{Possibilities and limits of public intervention aimed at LGBT+ people in Chile}

\author{
Caterine Galaz Valderrama' \\ Universidad de Chile, Chile. \\ Lelya Troncoso Pérez \\ Universidad de Chile, Chile.
}

\section{Cómo citar}

Galaz, C. y Troncoso, L. (2021). Posibilidades y límites de la intervención pública dirigida a personas LGBT+ en Chile. Propuestas Críticas en Trabajo Social - Critical Proposals in Social Work 1(2), 120 -142. DOI: 10.5354/2735-6620.2021.61580

\section{Resumen}

En los últimos años en Chile se han intensificado las demandas de colectivos LGBT+ y el Estado ha ido respondiendo reactivamente con algunas políticas e intervenciones públicas, pero desde lógicas que continúan siendo binarias y hetero-cis-normativas. Se presentan resultados de una investigación cualitativa

Palabras Clave: Intervención pública; $L G B T+$; diversidad sexual; políticas públicas; sexualidades. 
posibilidades y límites que se han articulado en las áreas de salud y educación, considerándolos como dos ámbitos relevantes en la intervención social. Se concluye que se ha extendido un ideal de "diversidad" dentro de la intervención que puede encubrir nuevas desigualdades y reificar nuevas posiciones de subordinación de las sexualidades no normativas, lo que puede contribuir a dar luces a un trabajo social más comprometido con estas luchas.

\section{Abstract}

In recent years in Chile, the demands of LGBT+ groups have intensified and the State has been responding reactively with some public policies and interventions, underpinned by an approach that continues to be binary and hetero-cis-normative. We present the results of qualitative research based on interviews with profes-

Keywords: Public intervention; $L G B T+$; sexual diversity; public policies; sexualities sionals from various public services and LGBT+ users of these services in three urban cities in Chile: Santiago, Concepción and Valparaíso. Among the main results are the conceptions of "sexual diversity" in the intervention with these groups, and the possibilities and limits that have been articulated in the areas of health and education, considering them as two relevant areas in social intervention. It is concluded that an ideal of "diversity" has been extended within the intervention that can cover up new inequalities and reify new positions of subordination of non-normative sexualities, which can contribute to shedding light on a social work more committed to these struggles.

\section{Introducción}

Desde el fin las dictaduras en Latinoamérica, el debate público respecto de los derechos de personas LGBT+ ha alcanzado una notoria visibilidad (Díez, 2013; Galaz et al., 2018), lo cual ha dado lugar a la creación de una serie de dispositivos jurídico-administrativos, estableciéndose una nueva categoría en el panorama de la gestión pública (Gauché, 2014): la diversidad sexual. En Chile, las actuaciones públicas referidas a las sexualidades no normativas se han dado por medio de indicaciones técnicas, protocolos, normas, algunas políticas y leyes (Galaz et al., 2018). Estas no se han establecido a partir de voluntades políticas, sino gracias a la demanda de diferentes movimientos sociales (Movimiento de Integración y Liberación Homosexual, Movilh, 2016).

Durante los años de la transición en Chile (1990-2005), la retórica del consenso y la reconciliación, a propósito del término de la dictadura, tuvo como uno de sus efectos 
la neutralización de la diferencia, obligando a la diversidad a ser "no-contradicción" (Richard, 2010). Durante los noventa se postergaron las demandas de los colectivos con identidades sexuales no heterosexuales (Rivas, 2011). De esta manera demandas como leyes de antidiscriminación, unión civil o matrimonio igualitario, de identidad de género, derechos sexuales y reproductivos o despenalización del aborto no fueron consideradas en un primer momento de la democracia.

Por otro lado, el mantenimiento de la sodomía como delito a través del artículo 365 del Código Penal, y la persecución y maltrato a las personas trans bajo el amparo del artículo 373, denominada Ley del Pudor, Moral y Buenas Costumbres (Garrido, 2015) dan cuenta de la criminalización de colectivos LGBT+ durante estos años. La ausencia de una voluntad política por ampliar las posibilidades del ejercicio de derechos de las personas LGBT+, no significó que las sexualidades quedaran excluidas de la intervención pública, sino que fueron abordadas en tanto "problemáticas". Así, se les controló sanitariamente por el riesgo del VIH/SIDA, acciones que más que información o prevención, buscaban proteger a la población de colectivos considerados como "peligrosos/as" para la sanidad nacional (Cabello, 2014).

Este período se ve atravesado por casos emblemáticos: en 2005, a propósito del juicio contra Karen Atala se instala en el debate público a las personas lesbianas dentro de los marcos de la familia (Cabello, 2014), atravesada fuertemente por la heteronormatividad y relacionado con su reconocimiento por parte del Estado como unidad básica de la sociedad. Desde 2011, las exigencias por matrimonio igualitario comienzan a ser más fuertes, instalándose como "una demanda principal de sociedades y culturas que buscan proponer una imagen sin discriminación y a favor de los derechos de los excluidos" (Cabello, 2014, p.19). Por otro lado, la muerte de Daniel Zamudio remece al país en 2012, lo que posibilita la aprobación del proyecto de ley contra la discriminación que plantea por primera vez sanciones para delitos por razones de discriminación. Finalmente, después de una intensa movilización social, en 2019 se aprueba la Ley de Identidad de Género que aún no tiene los efectos esperados de reconocimiento en los colectivos trans (Canales y Mallea, 2018).

Durante estos años el Estado chileno se ve presionado por la necesidad de visibilizarse como una nación moderna y respetuosa por los Derechos Humanos (Sabsay, 2011) buscando diversas estrategias para atender a la "agenda progresista" de la diversidad sexual que en otras partes del mundo ya comenzaba a instalarse (Sabsay, 2016). 


\section{Elementos teóricos: Estado, gubernamentalidad y personas LGBT+}

Para entender la intervención pública en el campo de las diversidades sexuales se debe comprender al Estado no como un ente supremo, autónomo, homogéneo y generador de ciertos órdenes sociales, sino más bien como parte integrante y cambiante de los diversos procesos históricos sociales por los que cruzan las sociedades. Esto implicaría pensar al Estado como proceso y no solo comprenderlo desde sus variantes institucionales y estructurales. En el fondo, el interés más que en la definición misma del Estado está puesto en el poder que este ejerce a través de una serie de mecanismos e instituciones. La tarea es visualizar el movimiento por el cual el Estado constituye, a través de diversas tecnologías móviles, un campo de verdad con objetos de saber; analizar las tecnologías de poder puestas en uso y sus efectos, más que las funciones que cumple el rol estatal (Bolívar, 2019).

El principio que sostiene el campo de la intervención estatal es la necesidad de asegurar cierta cohesión social interna y transformar situaciones que se entienden como problemáticas o de desigualdad entre sus miembros. Esta intervención estatal, materializada por ejemplo en diversas políticas públicas e intervenciones directas, no puede seguir pensándose en el tradicional binomio jerárquico gobernantes-gobernados/as, para comprenderla desde otro foco: no solo a partir de las medidas prácticas para solucionar un problema específico, sino en la maraña de significados, acciones y agentes involucrados que implementa (Lascoumes y Le Galés, 2012).

Consideraremos que las construcciones sociales que posibilitan la emergencia de ciertas categorías sociales como la llamada "diversidad sexual" se enmarcan en "matrices semiótico-materiales" (Estrada-Mesa, 2018; Hacking, 1999). Estas constituyen clasificaciones de los sujetos, establecen lo que es y no es un problema social atendible, determinan prácticas y limitan los repertorios de nominación que tienen efectos en la materialidad. Siguiendo a Bacchi y Goodman (2016) podemos afirmar que los modos en los cuales problemas sociales son representados se pueden abordar en sí mismo como intervenciones políticas que constituyen problemas políticos en diferentes contextos. En cierta manera, cuando se analiza la intervención pública se trata de desenmarañar, como diría Deleuze, las líneas de un dispositivo, sus curvas de visibilidad y sus curvas de enunciación. "Lo cierto es que los dispositivos... son máquinas para hacer ver y para hacer hablar” (Deleuze, 1990). 
Así, la intervención pública instala maneras de entender y actuar, que afectan directamente las trayectorias de las y los sujetos que consideran como centro de su actuación. A partir de la acción del Estado se establecen procedimientos y mecanismos por los que se determina qué personas quedan incluidas o excluidas de los sistemas. El dispositivo determina cómo se nombra y qué queda sin nombrarse.

Por ello, en este artículo nos centramos precisamente en analizar la intervención pública para ver cómo se despliegan esos regímenes de luz y enunciación del dispositivo -sobre el constructo de la "diversidad sexual"- y que deja en las penumbras.

Dicho despliegue se enmarca en relaciones de poder que resultan convenientes para determinados órdenes sociales, a través de la producción de regímenes de inteligibilidad que legitiman ciertas verdades frente a otras y en los cuales se vinculan las prácticas de gobierno y dominio de las poblaciones. Este conjunto de prácticas y operaciones de gobierno permiten constituir, definir y organizar las poblaciones, de tal modo que no sea necesario recurrir al ejercicio propio de la fuerza y la coerción del paradigma jurídico para ejercer poder (Foucault, 1975).

\section{Sujetos sociales y políticas identitarias}

Generalmente la articulación de políticas públicas sobre un tema tiene como condición de posibilidad la categorización en base a sujetos sociales concretos -en nuestro caso las personas LGBT+. De esta manera, el acceso a derechos se ve mediado por esta pertenencia identitaria, y por los modos en los cuales se ubica a las personas en relación con determinados repertorios discursivos, contextos temporales y geográfico (Ema -López, 2004). Por tanto, muchas de las políticas e intervenciones públicas responden a acciones sociales que evidencian un reconocimiento de derechos asociado a identidades determinadas, es decir, focalizado en categorías identitarias (Romero y Montenegro, 2018).

En este sentido, las llamadas políticas de la identidad -en las que se inscriben muchas de las políticas públicas, pero también de algunas luchas LGBT+, le dan una cierta centralidad a la consideración de "sujeto social”. Esto lleva, según Ema, a que la intervención pública sea una expresión de una cierta naturaleza anterior que requiere ser reconocida, ya sea a través de la accesibilidad de derechos iguales para todas las personas, o bien otorgando derechos específicos que se justifican a partir de una identidad diferencial. 
Algunas de las denominadas como políticas de la diferencia (o de la identidad) toman como punto de partida para sus demandas el reconocimiento y valoración de una identidad fijada y delimitada (pero ahora autodesignada y asumida como propia, no impuesta) como legitimación última de derechos específicos. (Ema-López, 2004,p.9)

En gran parte de las políticas públicas con enfoque identitario sigue primando una mirada esencialista de los sujetos, como entidades que dotan de significado a los procesos sociales al ser considerado como fuente y antecedente de la acción. Como plantea Butler, pensar que los sujetos pre-existen a la política, supondría

a) que la capacidad de acción solo puede establecerse recurriendo a un "yo" prediscursivo, aun cuando este se encuentre en medio de una convergencia discursiva, y $b$ ) que estar constituido por el discurso es estar determinado por él, donde la determinación cancela la posibilidad de acción. (Butler, 2001, p. 174).

Por tanto, que la intervención pública se enmarque en esta visión identitaria puede afectar las posibilidades de agencia del colectivo social, en la medida en que establece marcos de posibilidad para ser-hacer un "buen sujeto" de esa política -incluso si se ubica como resistencia a esas nominaciones. Ema López dirá que la contradicción, por tanto, "de esta posición esencialista radica en que fija, determina y obliga a los mismos sujetos que pretende representar y liberar" (2004, p. 9).

Desde perspectivas feministas interseccionales (Hill-Collins y Bilge, 2019) se ha problematizado también el riesgo de esencialismo en el desarrollo de proyectos y demandas colectivas, prestando atención a la potencial invisibilización de diferencias intragrupales, que necesita ser contrarrestada con nociones más complejas e interseccionales de comunidad y política. Esto se traduce en prestar atención a las relaciones de poder que producen las desigualdades sociales, sin desconocer que son particularmente grupos subordinados los que necesitan hacer usos estratégicos de políticas identitarias para avanzar en sus demandas particulares, lo que se ha denominado como "esencialismo estratégico".

\section{Apuntes metodológicos}

La presente investigación se adscribe a un enfoque sociocrítico de las ciencias sociales (Edwards y Potter, 1992; Garay et al., 2005; Gergen, 1996). Por ello, se llevó a cabo a través de una metodología de investigación cualitativa (Palumbo y Vacca, 2020) ya que 
se consideró la realidad social como un conjunto de relaciones interpretables entre sujetos (sujetos y objetos), en un contexto sociohistórico determinado. Cabe señalar que los métodos cualitativos de investigación apuntan a la comprensión de fenómenos en términos de sus significados y hacen referencia tanto a formas de aproximación al conocimiento como a las modalidades de análisis de este (Hammersley y Atkinson, 1994).

Para ello, se llevaron a cabo 40 entrevistas en profundidad y 6 grupos de discusión lo que permitió acceder a las dinámicas de relación entre las personas implicadas y al universo de significaciones de estos agentes en sus relaciones con el Estado, haciendo referencia a acciones pasadas o presentes. Los criterios de selección para personas interventoras sociales entrevistadas y participantes de los grupos de discusión fueron: a) que llevaran más de un año trabajando temáticas vinculadas a la "diversidad sexual", b) que trabajaran en algún dispositivo público (educación, salud, municipio, etc.) y c) que lo hicieran en los tres centros urbanos de la investigación (Santiago, Valparaíso y Concepción). En la selección de las personas usuarias se consideró que: a) se auto identificaran como LGBT+ (considerando variabilidad de cada identificación en los tres centros urbanos), b) que hayan asistido, en al menos una ocasión, a algún servicio público, y c) que fueran mayores de edad y residentes en las ciudades antes señaladas. Cada aplicación de instrumento en el campo contó con un consentimiento informado debidamente firmado por les participantes, y se garantizó el resguardo de la identidad y confidencialidad de los datos recabados (Villarroel, 2020).

Presentamos a continuación, los principales resultados de la investigación en tres categorías: a) las concepciones que se enarbolan sobre el constructo de la "diversidad sexual" en la intervención pública; b) las actuaciones en salud, como uno de los ámbitos sociales que emerge con más acciones directas en relación con este colectivo en Chile; y c) acciones en educación, como otra área de especial relevancia en relación con las vivencias LGBT+. Es importante remarcar que el trabajo de campo de esta investigación consideró diversos ámbitos de intervención pública, no obstante, la mayor cantidad de políticas y dispositivos consolidados en el tiempo desde la post dictadura a la fecha sobre la "diversidad sexual" se concentran en educación y salud. Por ello, en este artículo quisimos centrarnos en develar las lógicas de funcionamiento específicamente en estos campos. 


\section{Resultados: construcción problemática de la "diversidad sexual"}

En primer lugar, en las diferentes intervenciones públicas se puede visualizar la persistencia de una comprensión binaria y heteronormativa en torno a las sexualidades. Esto es, que generalmente las problemáticas que se enmarcan como "problemas a intervenir" (Galaz y Montenegro, 2015; Romero y Montenegro, 2018) vinculados a las sexualidades se comprende bajo la díada hombre/mujer o bien hetero/homosexual. Esto lleva a que se consideren prioritariamente acciones referidas a, por ejemplo, el embarazo adolescente o infecciones de transmisión sexual; la violencia en parejas heterosexuales, la defensa del derecho a la unión civil y/o matrimonio entre personas homosexuales, entre otras temáticas.

En el siguiente relato precisamente se da cuenta del heterosexismo (asumir que las personas son heterosexuales) y la heteronormatividad experimentada en la intervención social por personas LGBT+:

La intervención está marcada en general por la heteronorma. Desde el trato primero, se da por sentado, tú vas a preguntar algo y se da por sentado que tú eres hetero, o sea como que a nadie se le pasa por la cabeza que a lo mejor no. Salvo que sea algo como muy marcado, de fisonomía, la gente no se lo cuestiona. El concepto que se tiene de diversidad en ese sentido, es de anormalidad. Está el concepto de la heteronorma como lo normal, entonces ; ah, son distintos! hay que tratarlos distinto y todos somos distintos. $O$ sea, independiente que seas hetero o no, todos son distintos, cuando todos los hetero son distintos también. Pero eso es invisible. Siguen con una visión binaria en función de que, o sea las fichas de atención que llegan a los centros hablan de personas, o sea, hablan de género y hablan de identidades de género, pero no están bien desglosadas a nuestro parecer... revisamos estas orientaciones que llegaron, hicimos las observaciones a nivel regional y nacional, pero quedaron igual. Entonces, hay una idea binaria, así como también las identidades porque hacen la separación en trans, o sea en hablar de transfemenino o transmasculino y no hablas de trans no binario, entonces también eso, hay una falta de información y de conocimiento en profesionales. (Entrevista, interventora experta en género 7, Talcahuano)

Como se ve en esta misma cita anterior, en los últimos años, el avance en el reconocimiento social de personas transexuales y transgénero a nivel mundial, también ha tenido su correlato en la intervención pública cotidiana, generando diversas acciones en distintas áreas -legal, salud, educación, por ejemplo. El abordaje binario y heteronormado invisibiliza diferencias intragrupales, homogeneiza la diversidad sexual, y sobre enfatiza diferencias entre personas heterosexuales y comunidades LGBT+. 
A su vez, en algunos casos, se reproducen enfoques reduccionistas de la intervención al focalizarse solamente en algunos aspectos de las necesidades de personas trans (reconocimiento de nombre social), dejando otras necesidades que tensionan ámbitos estructurales menos visibles (como la inserción laboral). Esto se puede vincular también a que las demandas de movimientos LGBT+ se limitan muchas veces al reconocimiento social y la visibilidad cultural, descuidando aspectos estructurales de redistribución (por tanto, de derechos económicos, sociales y políticos de estos colectivos). Esto conlleva que muchas problemáticas de las personas LGBT+ queden en la opacidad de la intervención, sin actuaciones sostenidas en el tiempo y con temáticas invisibilizadas. Solo recientemente en algunas áreas emergen intervenciones enfocadas en problemáticas de personas trans como puede ser el acompañamiento en procesos de hormonación y tránsito identitario, o apoyo legal para cambio del nombre formal.

En la siguiente cita una cirujana explica que en la atención de personas trans en los últimos años, se deja poco margen a la autonomía de las personas, debido a miradas estereotipadas de los trans desde lo médico que se limitan a la permanencia del binario hombre-mujer y a la existencia de identidades fijas y estáticas:

Hay personas que hacen un tránsito corporal, hormonal, pero que no quieren hacer su adecuación genital, porque para ellos la condición de género no está vinculada a tener un pene o vagina. Aquí se respeta, pero en otros lados, no, se les insiste en transitar, en que tengan una identidad fija. La sociedad occidental le da demasiada importancia a ser mujer, ser hombre. Desde la temprana infancia, desde vestir a los niños con rosado, azul, celeste, desde la forma en cómo me han educado desde niña... creo que lo más importante es el individuo. Y si ese individuo se identifica como mujer, bien y si se identifica como hombre, bacán y si quiere hacer cosas de hombre que la sociedad dice que son de hombre y es mujer y la quiere hacer, bacán también. Pedir un tercer sexo para este tipo de pacientes es darle aún más importancia al tema del binarismo. (Profesional de salud, Santiago)

El aumento de colectivos sexuales disidentes ha permitido establecer alianzas estratégicas en la intervención social directa. En la investigación se evidenció cómo cada vez más ONG's y personas activistas reconocidas son involucradas en tanto "expertas". A estas no solo se les consulta sobre determinados temas, sino que se les invita a proyectos o programas de formación en diversas áreas, especialmente en salud y educación. En la siguiente cita, se enfatiza precisamente la necesidad de esta articulación sobre todo ante la falta de información y formación de profesionales:

Claro, es como un apostolado (leve risa), es más demandante porque tienes que 
reunirte, ponerte de acuerdo, transmitir información, nosotras estamos resistiendo y tratando de mantenernos como agrupación y luego meternos en espacios para sensibilizar, generar acuerdos con escuelas, con centros médicos.... difundir, formar... Es que, si no lo hacemos nosotras, pese a que trabajamos también, no lo hace nadie. Es un esfuerzo común pero necesario. (Grupo de discusión sexualidades disidentes, Santiago)

Aun cuando valoramos positivamente el involucramiento de las propias agrupaciones de diversidad o disidencia sexual en la capacitación de servicios sociales, es, a su vez, problemático que la responsabilidad sea depositada en las propias personas y colectivos LGBT+, y que como afirman, si no se hacen cargo "no lo hace nadie".

A su vez, se presentan tensiones y disputas entre los colectivos movilizados, particularmente respecto a grupos que han pasado a hegemonizar los espacios de intervención social en temáticas LGBT+. Como se ve en esta cita:

El problema es que siempre llegan a acuerdos y siempre aparecen los del X, organización de hombres gays, liderando, hablando y para la foto. Además, colocan sus temas y nuestros temas quedan muchas veces relegados. Por eso, decidimos armar estas mesas de trabajo porque queremos que se visibilicen otras temáticas y también que no siempre aparezcan los mismos hablando y tomando nuestra voz. (Grupo de discusión lesbianas y bisexuales, Talcahuano)

Se evidencian críticas recurrentes a cómo ciertos espacios de intervención son apropiados por algunos grupos y sus intereses particulares vinculados a experiencias e identidades específicas, en desmedro de otras necesidades y demandas LGBT+. Esto nos permite problematizar cómo bajo el paraguas "diversidad sexual" se pueden invisibilizar diferencias intragrupales, relaciones de poder, especificidades y multiplicidad de experiencias y necesidades. Como se señala en este extracto, el liderazgo de hombres gays opera en este caso desmedro de la visibilización de las necesidades de otras identidades sexuales y de género.

\section{La salud como ámbito de acogida y/o reproducción de diferencias}

El ámbito de salud surge como uno de los más demandados por las poblaciones LGBT+ en el país. Desde el retorno a la democracia, es una de las áreas donde más acciones públicas se han llevado a cabo para una mejor atención. Entre las últimas intervenciones destacan, por ejemplo, la creación de unidades específicas enfocadas en identidad 
de género en diversas regiones del país (Valparaíso, Concepción, en algunos centros hospitalarios de Santiago y Copiapó) en las que se atiende principalmente las necesidades quirúrgicas y solicitud de hormonación, apoyos ginecológicos y endocrinos, derivaciones de atención psicológica y psiquiátrica. Cabe señalar que, no obstante, estas recientes unidades no son parte de una política pública ministerial, sino que han nacido al amparo de profesionales sensibles a las dificultades trans, quienes han desarrollado en sus unidades hospitalarias presiones para que puedan instalarse como servicio.

En muchos casos, estas áreas no cuentan con presupuestos específicos y dependen de la donación horaria de otras unidades para que las y los profesionales puedan participar de las atenciones. De esta manera, se evidencia que la salud de personas LGBT+ más que una política pública, se va gestando como un asunto de sensibilidad profesional. La profesional entrevistada siguiente destaca precisamente lo difícil que ha sido instalar la problemática en ciertos centros públicos:
Es una encrucijada. Es que no es una enfermedad entonces cuesta instalar el tema en los servicios de salud considerando que no es una enfermedad. Pero sí es un derecho de las personas tener las prestaciones requeridas, y si las prestaciones que ellos necesitan son tratamientos hormonales que tienen que tener supervisión médica, que tienen que tener controles, chequeos, exámenes o alguna intervención quirúrgica para poder tener un proceso de tránsito de género como corresponde, entonces sí es una obligación del hospital que él pueda acceder a estas prestaciones. Es complejo pero sí, es un derecho de las personas porque no tengan una enferme- dad o algo de vida o muerte o algo así no significa que para esa persona no sea importante. Y también es un riesgo vital en el sentido de que, como te decía, los intentos de suicidio son tan comunes y tan altos que, si no se les da una oportunidad, un acceso a la salud esas personas terminan muriendo y mueren sin tener ninguna patología. (Entrevista profesional de salud, Santiago)

En los tres centros urbanos analizados, se presentan articulaciones entre el mundo activista y los centros de salud, posibilitando algunas campañas de difusión de derechos a nivel local. Ejemplo de ello es un caso en la región del Bío Bío, donde el vínculo entre personas trans, lesbianas y bisexuales y un Hospital de la zona, ha posibilitado el diseño y desarrollo de políticas de promoción de derechos. Así desde el activismo, con su presión por mayor reconocimiento, se consiguen instaurar políticas públicas "desde abajo", constituyéndose en la "Mesa activista LB" (lesbianas y bisexuales) y la "Mesa activista Trans" (personas transexuales, no binarias e intersex). Estas negociaron la conformación como una unidad de salud del hospital, llevándose a cabo, una serie de campañas internas y externas de sensibilización sobre los derechos de salud de personas LGBT+, pero también un protocolo de atención transversalizado al interior del hospital. 
Vimos que uno de los problemas que teníamos era la atención de salud (...) y llegamos a demandar al Hospital hasta que logramos un acuerdo hace poco. Hemos diseñado afiches que hemos colgado en los box de atención, hemos utilizado las instalaciones del Hospital. Cuando entregamos folletos informativos sobre cuidados para lesbianas, bisexuales y trans, hay personas que se enojan y se salen de sus casillas, porque en el fondo estamos ocupando un espacio público, en la difusión de cosas que a ellos no les gustan y que para ellos va contra la natura... ahí una ve $y$ vive ciertos encontrones, pero igual, una trata de ser lo más respetuosa posible y que si no quiere recibir la información está bien, pero que no es necesario armar un desmadre... pero seguimos ocupando ese espacio público. Logramos instaurar un protocolo de atención que ahora es obligatorio, y generamos cursos de formación permanente para profesionales y administrativos del Hospital. (Activista LGBT+, Entrevista personal Concepción, 30 de agosto 2018)

Pese a estas aperturas desde la intervención en salud, también al interior de la atención biomédica se destaca una reificación de las diferencias sexuales, en las que las personas LGBT+ emergen en una posición de subordinación respecto a las cisheterosexuales. Se rastrean argumentos respecto de la priorización de necesidades de salud en un sistema subsidiario público con escaso presupuesto, donde se enfatiza que las demandas LGBT+ no pueden tener un lugar privilegiado ya que existen otras necesidades de mayor importancia en cuanto a salud pública (se recalca particularmente las enfermedades terminales). Los sectores profesionales sensibilizados, pero minoritarios al interior del sistema, rebaten este argumento señalando que las demandas de salud de la población no heterosexuales deben ser consideradas como problema de salud pública y no como un asunto estético, donde se ha querido situar para aminorar su importancia y evitar posicionarlo como eje programático, tal como se condensa en la cita siguiente:

Nos apoyamos en nociones de respeto y equidad, si hay un paciente que puede ser incluido como parte de la diversidad, debe ser atendido como un derecho, con respeto, con nombre social, y de acuerdo a su propia orientación sexual o identidad de género; lo que suena súper sencillo, pero que no es fácil de trabajar al interior, porque hay resistencias de profesionales y no lo ven como un problema de salud pública. (Entrevista profesional de salud, Concepción)

Igualmente se destacan las resistencias de algunas personas profesionales al atender a la población LGBT+ por los prejuicios y estereotipos que se poseen. Algunas personas usuarias entrevistadas enfatizan que constantemente la primera atención suele ser desde una perspectiva cisheterosexual, lo que inhibe en muchos casos la adherencia a los 
tratamientos o la asistencia a centros médicos públicos; o bien, son tratadas a partir de los estereotipos existentes sobre las sexualidades disidentes lo que provoca deserción y rechazo al sistema de salud. Esto lleva a que las personas busquen soluciones y consejos en sus propias redes, fuera del sistema formal.

En general, se destaca la prevalencia de un enfoque binario y biologicista en la práctica de atención directa, denunciando falta de formación profesional en la variabilidad sexual y sus necesidades específicas. El uso de etiquetas estereotipadas de las sexualidades se relaciona también con objeciones morales presentes en profesionales de salud, que desde lógicas conservadoras consideran a estos colectivos como desviados o fuera de la norma social.

Es que te tratan como heterosexual desde el inicio, eso inhibe a las mujeres lesbianas, no binarias o bisexuales que puedan acudir, porque no se sienten acogidas y van una vez y no siguen yendo... otras no siguen yendo porque al contar su orientación o identidad, solo reciben de respuesta prejuicios, o violencias directamente, porque algunas son tratadas mal como bichas raras, entonces no siguen yendo... al final la gente se va pasando información informalmente o por las redes entre nosotras. (Grupo de discusión, Concepción)

\section{Posibilidades y límites en educación}

En Chile existe desde el año 2012 la ley antidiscriminación y del 2017 una circular con orientaciones para la integración de la niñez trans, pero ambos cuerpos normativos no llegan a implementarse de manera transversal en las escuelas públicas y/o privadas ${ }^{2}$.

Existe, sin embargo, una distancia entre las normativas y su implementación a nivel cotidiano: por un lado, se debe al principio de la libertad de enseñanza, justificativo que algunos establecimientos esgrimen para obstaculizar el desarrollo de una educación sexual a sus alumnos/as y de atención a todas las sexualidades. También se destaca la falta de conocimientos de los equipos directivos y profesorado respecto a cómo traducir metodológicamente una normativa a acciones concretas al interior de los centros, sin recaer en estereotipos problemáticos, teniendo en cuenta el vacío formativo declarado en sus trayectorias profesionales en temática de sexualidades.

Constantemente emerge en las escuelas un "no sabemos qué hacer", sobre todo, ante la mayor visibilización, presión y reivindicación desde el estudiantado de orientaciones

\footnotetext{
2 Desde el año 2019 rige la Ley de Identidad de Género, pero el trabajo de campo de esta investigación se llevó a cabo cuando estaba aún en discusión parlamentaria.
} 
e identidades disidentes. En la siguiente cita se destaca que es difícil llegar a sectores del profesorado más reacio a la apertura en estas temáticas, ya que la formación es de libre elección:

El problema que tuvimos es que la apuesta del ministerio fue capacitación de profesores sin ninguna vinculación con las instituciones escolares. Se capacita al profesor que quería capacitarse. No llega a los que no están sensibilizados. Pero un profesor por su cuenta, si no tiene el apoyo o el interés de la escuela, de la comunidad en general, lo que logra hacer es muy poco. Entonces, ahí hubo una apuesta con poca voluntad política (...) luego lo que hizo Piñera después es de las peores cosas: mercantilizar la educación sexual ya que cada colegio si tiene plata contrata y además contrata lo que quiere. Lo que hizo fue darles una responsabilidad a los colegios, eran las escuelas las que estaban convocadas a contratar, a tener un programa, a hacer una apuesta ideológica. Se trabajó con lógicas del currículum emergente lo que no solo es timorato en términos de que no están haciendo algo mínimo que decís “jvamos a hacer estas definiciones sobre la sexualidad!” sino que además es en exceso optimista si es muy dificil que el profesor por su cuenta se ponga a diseñar unidades de aprendizaje. Porque no tienen tiempo para hacerlo, porque no tienen las competencias”. (Profesional de la educación, Entrevista personal Santiago, 12 de junio de 2018)

Por otra parte, en el ámbito educativo vuelven a presentarse las objeciones morales de parte de profesionales. En ese sentido, se destaca también el carácter ideológico que han tenido las diversas directrices ministeriales de acuerdo con los gobiernos de turno en democracia y su apertura o rechazo a las disidencias sexuales, como destaca la siguiente profesional:

Es que también podemos llegar a alianzas con ciertas escuelas, y en otras no nos dejan entrar. El año pasado en algunas que hicimos talleres en conjunto con los servicios de salud, nos llegaron reclamos de padres y nos pidieron avisar a las escuelas antes, para no enviar a sus hijos. pero ese rechazo también se ven en profesores/as y en algunos directores que te ponen mil excusas, pero al final ves que es porque no quieren, porque son religiosos o son conservadores...es difícil. (Grupo de discusión profesionales, Santiago)

Aun siendo así, desde los años 2000 se han ido diseñando e implementando algunas actuaciones respecto de la educación sexual y su vinculación con las vivencias LGBT+. No obstante, estas pueden estar limitadas por factores estructurales, como la posibilidad de acomodación de este tipo de formación al propio proyecto educativo que tienen las escuelas, y el disponer o no de recursos económicos para acceder a servicios específicos 
formativos (sobre todo en la educación privada) o acceder a servicios gratuitos (en escuelas públicas). A su vez, se destaca que mayormente la formación en sexualidades es débil y tangencial y que no se valora como un aspecto imprescindible a ser incorporado en el currículum escolar.

Cuando se incorpora persiste mayoritariamente un enfoque fuertemente biologicista y heteronormado en la formación que reciben les estudiantes y educadores: es decir, se centran en temáticas como la prevención de embarazo adolescente y la transmisión de enfermedades de transmisión sexual, y escasamente se hace alusión a otras sexualidades, a afectividad y el deseo en sus múltiples posibilidades y vivencias, como enfatiza el siguiente experto:

Nosotros tenemos elementos de la sexualidad en el currículum, en dos asignaturas, en orientación y en ciencias naturales. Pero es muy precario en esta materia, tiene que ver con cómo manejar los afectos en el caso de orientación, es un currículo conservador. $Y$ en el de ciencias naturales, fundamentalmente cuestiones que tienen que ver con iniciación sexual, con anatomía, cosas así, un currículum bastante heterocentrista, porque tiene que ver con cómo hacer para no embarazarse o no transmitirse enfermedades (...) El año pasado hicimos una revisión curricular y una comparación con estándares internacionales de educación sexual. Y lo que nos encontramos es que en Chile se aborda un $34 \%$ de los estándares internacionales y ese $34 \%$ eran algunas cosas sobre los afectos, y después lo que tiene que ver con orientación sexual en biología con los más grandes. Pero otros elementos, que tiene que ver más con los elementos culturales sobre la sexualidad, están al debe (Profesional Ministerio de Educación, Entrevista personal Santiago, 17 de mayo 2018)

En algunos centros educativos se enfatiza que en los últimos años se han realizado talleres para la comprensión de sexualidades disidentes y se han ido incorporando algunas actuaciones hacia las poblaciones LGBT+, como puede ser el disponer de baños no binarios, posibilidades de modificación o selección del uniforme escolar de acuerdo con la orientación/identidad del estudiantado o el uso del nombre social. Sin embargo, así como estas iniciativas pueden considerarse como signos de apertura hacia algunas vivencias no heterosexuales, a la vez, se denota un tratamiento poco complejo respecto de las desigualdades estructurales en que se enmarcan estas trayectorias, reduciéndose a aspectos materiales específicos que, siendo necesarios, se posicionan como si fuera lo único a atender, dejando en suspenso otros aspectos como la prevención y erradicación de violencias dirigidas contra población LGBT+ que vehiculiza la dinámica escolar a múltiples niveles. 
Casi nada en términos de homo/lesbo/transfobias, yo diría que es prácticamente nada... siempre es el tema más de los cuidados de enfermedades de transmisión sexual o de embarazo adolescente, lo típico, pero es difícil hasta distribuir condones como te decía, resistencias de las escuelas y de las familias, pero algunas escuelas empiezan a abrirse, aunque también con harto estereotipo también... terminan haciendo difusión de que pusieron baños o sobre todo el uso del nombre, pero algo más integral, no, es difícil. (Grupo de discusión profesionales, Santiago)

De esta manera, las medidas adoptadas siguen siendo limitadas tanto a la hora de abordar formas de violencia, discriminación que afectan a población LGBT+, como a la hora de desarrollar perspectivas más amplias y complejas, que permitan, por ejemplo, visibilizar la heternormatividad como un sistema de poder que impacta en las vidas de todas las personas, no solo las "diversas", pero con efectos diferenciados. Lo que sin duda se deja sin atender es el reto de desheterosexualizar la educación y la pedagogía (flores, 2017). En el ámbito educacional tienen mucho que aportar las pedagogías disidentes sexuales, que por ejemplo, se han enfocado en "la producción de heteronormatividad desde la ignorancia, a través de los modos en que la escuela restringe ciertos sujetos, corporalidades y formas de enunciar los deseos que se consideran desviados e imposibles" (Troncoso et al., 2019). En ese sentido es importante reconocer que la comunidad LGBT+ ha aportado no solo con demandas y activismos, sino a su vez con saberes y apuestas pedagógicas que suelen ser ignoradas en la formación pedagógica general y que podrían aportar mucho a la formación de educadores que insisten en "no saber qué hacer", para que cuenten con herramientas para reconocer, cuestionar y enfrentar la hétero y cisnormatividad en el sistema y las políticas educacionales.

\section{Conclusiones}

A pesar de numerosos avances en la atención y reconocimiento de población LGBT+ en Chile, permanecen múltiples problemáticas y tensiones; lo que alumbra vías críticas para llevar a cabo un Trabajo social más pertinente. Por un lado, se sigue interviniendo bajo una lógica que posiciona el "problema" y la responsabilidad de solucionar problemas en las personas y colectivos LGBT+, sin cuestionar las dimensiones más estructurales y sistémicas de reproducción de la cisheteronormatividad. Esto conlleva un abordaje de la diversidad como variación benigna que invisibiliza relaciones de poder asumiendo un armónico abanico de sujetos diversos (McKinzie y Richards, 2019). 
Teniendo esto como horizonte, desde un Trabajo social crítico (González-Saibene, 2021; 2015) se pueden tensionar el diseño de políticas e intervenciones altamente identitarias, problematizando sus dimensiones esencialistas y homogeneizantes, para dar un giro que permita desentrañar esas condiciones estructurales y contextuales que dificultan precisamente las trayectorias de reconocimientos más complejos de estos colectivos. González-Saibene (2021) -al hacer una re-lectura de la ruptura que significó la reconceptualización para el Trabajo Social- enfatiza la importancia de posturas epistémicas críticas en la disciplina para poder acompañar desde intervenciones "fundadas" a las poblaciones subalternizadas en sus ejercicios de derecho y ciudadanía. Incluso hace más de 20 años, Dominelli y MacLeod, llamaron a fomentar un "Trabajo social feminista", que trabajara no sólo con los individuos de forma aislada, sino que se planteara desde enfoques transformadores que considerara las matrices de desigualdad y los contextos (Dominelli y MacLeod, 1999).

Como señalamos anteriormente, la intervención pública que muchos/as trabajadores sociales reproducen, es limitante cuando se articula como un dispositivo identitario (Romero y Montenegro, 2018) que establece qué ver, qué atender y cómo nombrar, invisibilizando diferencias intragrupales o reproduciendo representaciones estereotipadas de lesbianas, gays o personas trans, perdiendo de vista cómo se producen las diferencias en medio de relaciones de poder y jerarquías que producen desigualdades. La práctica de un trabajo social feminista, según Wendt y Moulding (2016) permite transformar las formas tradicionales en que el trabajo social ha considerado tanto al género como a las sexualidades, para poner énfasis en las relaciones de poder que sustentan a estas nociones.

Ese ejercicio del Trabajo Social frente a la política social, corre el riesgo de reificar y reproducir las desigualdades estructurales. Esa construcción de sujeto a priori que antecede a la intervención misma -al aglutinar las necesidades en un todo nebuloso llamado "diversidad sexual"- tiene como efectos la homogeneización de las necesidades particulares y de las violencias que puedan experimentar las diversidades y disidencias sexuales. Un Trabajo social crítico desde perspectivas interseccionales puede tensionar estas configuraciones, dadas por sentado en la lógica de la intervención, llevando el cuestionamiento a las condiciones estructurales, para prestar atención a cómo experiencias particulares de exclusión, marginación y opresión se materializan en medio de sistemas y estructuras articuladas de poder (Troncoso et al., 2019). Desestabilizar abordajes identitarios esencialistas y reduccionistas es necesario para posibilitar otras y múltiples formas de vida, experiencias y promover una lectura más aguda y compleja 
de las condiciones contextuales en las cuales se sitúan las experiencias cotidianas de personas LGTBI+. De hecho, esto se consigna como parte de la misión de la disciplina: en la definición global del Trabajo Social de la Federación internacional de Trabajadores/a sociales (2014) se plantea que son fundamentales las reflexiones respecto de las diversas fuentes de opresión y/o privilegio, basadas en diferencias como el género, la orientación sexual, la clase entre otras, y establecer estrategias de acción que aborden no sólo problemas individuales sino estructurales.

A su vez, en el campo de la intervención social suelen primar muchas veces las voluntades personales por sobre las actuaciones políticas programáticas, para asegurar una atención digna y respetuosa de la población LGBT+. Junto a lo anterior se visibiliza una crítica recurrente a la falta de formación actualizada y crítica en temas de género y sexualidades de profesionales tanto de la educación, salud, como de otras áreas, siendo los propios colectivos LGBT+ los que tienen que asumir, muchas veces sin remuneración, esta labor formativa como parte de su activismo. Esto devela no solo la necesidad de una política pública transversal -ausente hasta ahora- que garantice derechos a la población LGTB+ sino también la importancia de que ese proceso sea participativo y vinculante en relación directa con los colectivos sociales (políticas que sean hechas desde abajo), de manera de asegurar un proceso que sea más pertinente (Silva et al., 2020)

En relación con lo antes mencionado, continúa siendo muy difícil avanzar a abordajes más integrados e interseccionales (Hill-Collins y Bilge, 2019; Troncoso et al., 2019) en el Trabajo Social, que permitan visibilizar cómo se articulan entre sí diversas estructuras de poder (heteronorma, patriarcado, neoliberalismo, etc.) impactando de diferentes modos en las experiencias y condiciones de vida materiales de las personas y colectivos LGBT+ que se sitúan a su vez en diversos contextos históricos, sociales y económicos. En ese sentido, un trabajo social crítico implica desentrañar los mecanismos en que se producen las distintas categorías desde las cuales se comprende a los sujetos, las lógicas excluyentes y los efectos de poder que conllevan, pero sin obviar estas categorías, sino abordando las maneras en que estas establecen cierto orden social normalizado a través de prácticas institucionales. Como señala Romero y Montenegro (2018) se requiere un análisis interseccional precisamente para evitar que estas categorías sean vistas como neutrales y aproblemáticas.

Finalmente, señalar que para avanzar en una adecuada atención a las necesidades de población LGBT+ en Chile es urgente trabajar paralelamente en diferentes ámbitos para: incorporar y valorar debidamente los saberes sobre géneros y sexualidades en la 
educación (en particular saberes construidos por personas y comunidades LGBT+); reconocer las diversidades y disidencias sexuales en todo ámbito de relaciones humanas y políticas públicas; avanzar en la despatologización y evitar la fetichización de sexualidades e identidades de género diversas; y resistir a la despolitización de la diversidad que la hace aparecer como mera variación benigna de diferencias invisibilizando en su abordaje las relaciones y desigualdades de poder (en sus dimensiones tanto estructurales, como sistémicas e interpersonales), que se materializan en las experiencias de exclusión, discriminación, opresión y violencia que necesitamos erradicar.

Como destaca María Eugenia Hermida (2020), nos encontramos en una tercera interrupción del Trabajo social que implica descolonizarlo y despatriarcalizarlo, pero no sólo en función de prácticas orientadas hacia "afuera", sino revisitando las propias formas de ver y hacer de la disciplina. La autora concluye que urge "irrumpir en las categorías que nos impiden pensar, para poder ver esas experiencias otras que habilitan la justicia social y una vida digna" (p.116).

\section{Referencias bibliográficas}

Bacchi, C. y Goodwin, S. (2016). Poststructural Policy Analysis. A Guide practice. Palgrave McMillan.

Bolivar, M. (2019) Crisis de legitimidad del estado contemporáneo. Replanteando el papel de la ética pública. Polis (México), 15, 33-63.

Butler, J. (2001). Mecanismos psíquicos del poder: teorías sobre la sujeción (Vol. 68). Ediciones Cátedra.

Cabello, C. (2014). Ciudadano gay. Visualidades y plusvalía sexual en tiempos liberales. [Tesis de Magíster en Comunicación política, Universidad de Chile].

Canales, V. y Mallea, R. (2018). Estudio de la identidad de género en Chile a la luz de la acción de no discriminación arbitraria (Ley 20.609) y el derecho a la identidad. Anuario de Derechos humanos, 14, 129-140. DOI: 10.5354/0718-2279.2018.49408

Deleuze, G. (1990). ¿Qué es un dispositivo? En B. Gots, H. L. Dreyufis, M. Frank, A. Gluchsmann y E. Balibar (eds.), Michel Foucault, filósofo. Gedisa. 
Díez, J. (2013). Explaining Policy Outcomes: The Adoption of Same-Sex Unions in Buenos Aires and Mexico City. Comparative Political Studies, 46(2), 212-235.

Edwards, D. y Potter, J. (1992). Discursive Psychology. SAGE Publications.

Ema-López, J.E. (2004). Del sujeto a la agencia (a través de lo político). Athenea Digital: Revista de pensamiento e investigación social, 5, 1-24.

Estrada-Mesa, D. (2018). ¿Para qué sirve una filosofía de los dispositivos? Eidos, 29. https://doi.org/10.14482/eidos.29.9315

Dominelli, L. y McLeod, E. (1999). Trabajo social feminista. Cátedra.

flores, v. (2017). ESI: Esa Sexualidad Ingobernable. El reto de des-heterosexualizar la pedagogía, en Tropismos de la disidencia (pp. 147-158). Palinodia.

Federación internacional de Trabajadores/as sociales. (2014). Definición global del Trabajo Social. Reunión General de la IFSW y la Asamblea General de la IASSW en julio de 2014. https://www.ifsw.org/what-is-social-work/global-definition-of-social-work/

Foucault. M. (1975). Defender la sociedad. FCE.

Galaz Valderrama, C., Sepúlveda Galeas, M., Poblete Melis, R., Troncoso Pérez, L., y Morrison Jara, R. (2018). Derechos LGTBI en Chile: Tensiones en la constitución de otredades sexualizadas. Psicoperspectivas, 17(1).

https://doi.org/10.5027/psicoperspectivas-vol17-issue1-fulltext-1165

Galaz, C. y Montenegro, M. (2015). Gubernamentalidad y relaciones de inclusión/ exclusión: los dispositivos de intervención social dirigidos a mujeres inmigradas en España. Universitas Psychologica, 14(5), 1667-1680.

https://dialnet.unirioja.es/servlet/articulo?codigo $=5716436$

Garay, A., Iñiguez, L. y Martínez, L. (2005). La perspectiva discursiva en psicología social. Subjetividad y Procesos Cognitivos, 7, 105-130.

Gauché, X. (2014). Análisis crítico de la Ley 20.609, que establece medidas contra la discriminación, a la luz del derecho internacional de los derechos humanos y las convenciones de la OEA sobre discriminación de 2013. Revista Chilena de Derecho y Ciencia Política, 5(1), 11-58. 
Garrido, J. C. (2015). Diversidades en la transición: Homofobia y el movimiento LGBT en Chile, 1990-2000. Historias que vienen: Revista de Estudiantes de Historia, 6.

Gergen, K. (1996). Realidades y relaciones. Aproximaciones a la construcción social. Paidós.

González-Saibene, A. (2015). Acerca de La Intervención / About Procedure. R evista Rumbos TS. Un Espacio Crítico Para La Reflexión En Ciencias Sociales, 11, 22-40.

http://revistafacso.ucentral.cl/index.php/rumbos/article/view/82

González-Saibene, A. (2021). El impacto de las producciones filosóficas y teórico/epistemológicas en la constitución de la disciplina. Propuestas Críticas en Trabajo Social - Critical Proposals in Social Work, 1(1), 101-122. DOI: 10.5354/2735-6620.2021.61238

Hacking, I. (1999). The Social Construction of What? Harvard University Press.

Hermida, M. E. (2020) La tercera interrupción en Trabajo Social: descolonizar y despatriarcalizar. Revista Libertas, 20(1), 94-119. DOI: 10.34019/1980-8518.2020.v20.3053494

Hammersley, M. y Atkinson, P. (1994). Etnografía: métodos de investigación. Paidós.

Hill-Collins, P. y Bilge, S. (2019). Interseccionalidad. Editorial Morata.

Lascoumes, P. y Le Galès, P. (2004). Introduction: L'action publique saisie par ses instruments. En , Gouverner par les instruments (pp. 11-44). Presses de la Fondation Nationale des Sciences Politiques.

Lascoumes, P. y Le Galès, P. (2012). Sociologie de l'action publique (2e édition). Editorial Armand Colin.

McKinzie A.E. y Richards P.L. (2019). An argument for context-driven intersectionality. Sociology Compass, 13(4). Artículo e12671. https://doi.org/10.1111/soc4.12671

Movimiento de Integración y Liberación Homosexual. (2016). XIV Informe Anual: Derechos Humanos de la Diversidad Sexual Chilena (Hechos 2015). http://www.movilh.cl/documentacion/2016/informe/XIV-Informe-de-DDHH-2015.pdf

Palumbo, M., y Vacca, L. (2020). Epistemologías y metodologías críticas en Ciencias Sociales: precisiones conceptuales en clave latinoamericana.Revista Latinoamericana De Metodología De Las Ciencias Sociales (Relmecs), 10(2). Artículo e076. https://doi.org/10.24215/18537863e076 
Richard, N. (2010). Crítica de la Memoria (1990-2010). Universidad Diego Portales.

Rivas, F. (2011). De la homosexualidad de Estado a la Disidencia Sexual: Políticas sexuales y postdictadura en Chile. Texto presentado en Seminario Tercer Circuito de Disidencia Sexual: No hay respeto, organizado por Colectivo Universitario de Disidencia Sexual, CUDS. Universidad de Chile.

https://www.bibliotecafragmentada.org/wp-content/uploads/2013/03/De-la-homosexualidad-de-Estado-a-la-Disidencia-Sexual.pdf

Romero Bachiller, C. y Montenegro, M. (2018). Políticas públicas para la gestión de la diversidad sexual y de género: Un análisis interseccional. Psicoperspectivas, 17(1), 64-77. https://dx.doi.org/10.5027/psicoperspectivas-vol17-issue1-fulltext-1211

Sabsay, L. (2011). Fronteras sexuales: Espacio urbano, cuerpos y ciudadanía. Paidós

Sabsay, L. (2016). The political imaginary of sexual freedom: subjectivity and power in the new sexual democratic turn. Studies in the Psychosocial. Palgrave Macmilan.

Silva, R., De los Reyes, P. y Galaz, C. (2020). Cuerpos diversos e inclusión (in)subordinada. Una propuesta de políticas urbanas interseccionales. Serie Domesticar la Ciudad. Vicerrectoría de Investigación y Desarrollo de la Universidad de Chile.

Troncoso, L., Follegati, L. y Stutzin, V. (2019). Más allá de una educación no sexista: aportes de pedagogías feministas interseccionales, Pensamiento Educativo. Revista de Investigación Educacional Latinoamericana 2019, 56(1), 1-15. doi: 10.7764/PEL.56.1.2019.1

Villarroel, R. (2020). Caracterización diferencial de la investigación social. Revista Ethika, 1. DOI: $10.5354 / 2452-6037.2020 .57085$

Wendt, S. y Moulding, N. (2016). Contemporary Feminisms in Social Work Practice. Routledge. 


\section{Agradecimientos}

Fondecyt Regular N 1170417, ANID-CONICYT, “Acción pública y diversidad sexual en Chile: Construcciones sociales en democracia (1990-2016)". Investigadora responsable: Caterine Galaz Valderrama.

\section{Biografía de las autoras}

Caterine Galaz Valderrama, Académica del Departamento de Trabajo Social, Facultad de Ciencias Sociales de la Universidad de Chile. Doctora y Magíster en Ciencias de la Educación de la Universidad Autónoma de Barcelona, Magíster en Políticas Sociales de la Universidad ARCIS, y Licenciada en Comunicación Social de la Universidad de Santiago de Chile. Reside en Chile. Co-Coordinadora del Núcleo Diversidad y Género: Abordajes feministas interseccionales del Departamento de Trabajo Social de la Universidad de Chile. Co-coordinadora de la Red de Interseccionalidad, género y prácticas de resistencias de la Universidad de Chile, coordinadora de la línea de Resistencias feministas del Grupo de Trabajo Memoria y prácticas de resistencias (CLACSO). Coordinadora del Grupo de Trabajo de CLACSO Migraciones Sur-Sur.

Correo electrónico: cgalazvalderrama@uchile.cl

ORCID ID: http://orcid.org/0000-0001-6301-7609 https://uchilefau.academia.edu/CaterineJoannaGalaz

Lelya Troncoso Pérez, Psicóloga Social Feminista, es Licenciada en Psicología de la Universidad de Valparaíso (Chile), Master en Psicología Social de la Universidad Autónoma de Barcelona (España), Master en Estudios de Género de la Universidad de Lund (Suecia) y Doctora en Psicología de la Universidad de Chile. Actualmente es profesora asistente del Departamento de Trabajo Social de la Universidad de Chile. Sus ámbitos de docencia e investigación son la psicología social crítica, los estudios feministas, las teorías críticas de sexualidad, interseccionalidad, memoria y derechos humanos. Es co-coordinadora del Núcleo Diversidad y Género: abordajes feministas interseccionales del Departamento de Trabajo Social de la Universidad de Chile. Integrante del Grupo de Estudios Género y raza: miradas interseccionales. Integrante del Programa de Psicología Social de la Memoria.

Correo electrónico: lelyatroncoso@uchile.cl

ORCID ID: https://orcid.org/0000-0002-9197-3611 https://uchile.academia.edu/LelyaPérez 